\title{
GPPS-TC-2019-0055
}

\section{Computational and experimental investigation of mechanical stresses induced by rotor-stator interaction in an industrial centrifugal compressor}

\author{
Holger Franz \\ MAN Energy Solutions SE \\ holger.franz@man-es.com \\ Oberhausen, Germany
}

\author{
Sebastian Legrand \\ MAN Energy Solutions SE \\ sebastian.legrand@man-es.com \\ Oberhausen, Germany
}

\author{
Tobias Wiedemann \\ MAN Energy Solutions SE \\ tobias.wiedemann@man-es.com \\ Oberhausen, Germany
}

\begin{abstract}
A tool chain for the numerical prediction of high cycle fatigue (HCF) in an industrial centrifugal compressor during resonant stimulation, caused by rotor-stator interactions, is set up and validated against experimental investigations. The compressor under investigation is built up of an inlet guide vane (IGV), an open impeller, a vaned diffuser and an outlet volute. This type of centrifugal compressor is typically used in integrally geared compressors. The experimental investigation was performed on an open loop test rig of MAN in Oberhausen. Frequency domain based CFD simulations were used to investigate the fluid-structure-interactions. The resulting forced response alternating stresses were examined at a cyclic symmetric model by the use of a FE analysis.

Two nodal diameters of one particular eigenmode are investigated. Once the excitation is caused by the diffuser and the other time by the IGV. The aerodynamic excitation and damping forces are changing along a speed line from choke to surge. Therefore, three different operating conditions are experimentally and numerically investigated in detail for each resonance. The numerically predicted alternating stresses occurring in the tuned system are compared directly to measurements with strain gauges on the impeller blade and to non-intrusive tip timing probe data.

Objective of the paper is to show that the numerical tool chain is able to predict the alternating stresses due to rotorstator-interaction correctly. Especially, the simultaneous calculation of excitation and damping in one frequency domain based CFD simulation is not commonly used. Although the magnitude of the predictions and the measurements show some offset, the qualitative validity of the numerical prediction could be proved. This enables a quick evaluation of critical operating conditions. Systematic evaluations of the aerodynamic excitation and damping mechanisms are conducted. These analyses improve the understanding of the damaging potential of HCF due to different excitation sources.
\end{abstract}

\section{INTRODUCTION}

Rotor-stator-interactions can cause high unsteady pressure fluctuations on the stator and rotor blade-rows of a centrifugal compressor stage. If the frequency of these pressure fluctuations is equal to an eigenfrequency of a bladerow, this blade-row is stimulated in resonance. The resulting vibration amplitude of the blades causes high alternating stresses within in the material, which can lead to a large high cycle fatigue (HCF) of the blades. The magnitude of the induced alternating stresses is depending on the material properties, the excitation force and the damping of the system. Equation (1) gives the general equation of motion for a single degree of freedom system [e.g. (Platzer \& Carta, 1988)]

$$
[M] \underline{\ddot{s}}+[C] \underline{\dot{s}}+[K] \underline{\mathrm{s}}=f_{\text {aero,exc }}
$$

$[\mathrm{M}]$ is the mass and $[\mathrm{K}]$ the stiffness matrix. The damping matrix [C] consists of the mechanical, the material and the aerodynamic damping. As the impeller is manufactured of one piece, no mechanical damping is existing. The consensus which will be confirmed later - is that the aerodynamic damping is approximately an order of magnitude greater than the material damping and thus, this contribution is nearly negligible. The aerodynamic excitation force is standing on the right-hand side of equation (1).

The compressor stage under investigation consists of an IGV, an open impeller, a vaned diffuser and an outlet volute. Typically, the compressor stage is utilized in integrally geared compressors and running on a fixed speed. Thus, with regard to the frequencies of pressure fluctuations induced by rotorstator- interactions, in most cases a resonant free steady operation of the compressor is possible. But even in these alleged resonant free compressor designs excitation of eigenmodes can occur, if unsteady phenomena like rotating stall arise with the corresponding frequencies, c.f. (Jenny \& Bidaut, 2016), (Chen, et al., 2017), (Wagner, et al., 2011). Within this paper these phenomena are not discussed, but 
(Mischo, et al., 2018) shows that this issue is under investigation. Other excitation sources can be installations, e.g. pipe bends or struts, upstream or downstream the compressor.

The aerodynamic design of integrally geared compressors is often highly customized and a single-item production. Additionally, a special issue of industrial centrifugal compressor stages is, that the very same stage can be operated at varying inlet conditions and gas compositions, c.f. (Fruth, et al., 2016). Thus, extensive HCF investigations during the design of a compressor, as it is usual in series productions like aero engines (Srinivasan, 1997), (Seinturier, et al., 2004), (Seinturier, 2007), usually are not conducted. Resonant crossings due to rotor-stator interactions can occur in the first draft of a tailormade machine. Either these crossings can be avoided by a redesign, or the crossings have to be assessed in terms of HCF. This is the purpose of the presented tool chain.

To numerically resolve the unsteady pressure fields, it is possible to conduct a CFD simulation in the frequency domain. Then, only the frequencies of interest are considered, which, in comparison to URANS simulations, significantly reduces the time effort for the computations. The results of both methods are in good accordance to each other, c.f. (Elder, et al., 2013). In this investigation the non-linear harmonic (NLH) approach is used to simulate the unsteady flow (Debrabandere, et al., 2012), (Debrabandere, et al., 2013).

Rotor-stator-interactions in the context to fluid-structureinteractions (FSI) have been investigated by several authors. (Lerche, et al., 2012) showed a centrifugal compressor stage, which was damaged by HCF. He was able to redesign the impeller to a more robust geometry by the use of numerical FSI calculations. In two publications Zemp investigated the mechanism of rotor-stator interactions causing blade vibrations. In his first investigation, the influence of inlet distortions was analyzed ( (Zemp, et al., 2011), (Zemp, et al., 2011)). One of the main conclusions he draws, was that the unsteady pressure field at impeller leading edge is very sensible to changes in incidence angle. The second investigation deals with the excitation mechanism due to impeller-diffuser-interaction (Zemp \& Abhari, 2012). A variation of the vaneless space between the impeller and the diffuser vanes was conducted. An important finding in the context to this paper is that excitability of the structure is mode dependent and thus, blade vibration analysis must be performed at realistic operating conditions.

A very substantial experimental investigation was conducted by Kammerer (c.f. (Kammerer \& Abhari, 2010), (Kammerer \& Abhari, 2010)). He stimulated a centrifugal compressor in resonance by the use of inlet distortion screens, which are comparable to an IGV. He points out, that the aerodynamic damping, which is induced by the vibration of the blades, is the most important damping mechanism in centrifugal compressors. Thereby, the distribution of the pressure fluctuations, which cause the aerodynamic damping, can be dependent on the volume flow of the compressors. This behavior will be shown within this paper.

Especially, with focus on centrifugal compressors with vaned diffuser and volute, Dickmann (Dickmann, et al., 2005),
(Dickmann, et al., 2009) could show an influence of the diffuser-volute-interaction onto the impeller vibration. This effect is not analyzed in this investigation. To reduce the effort of the CFD calculation, the volute is not part of the numerical domain.

\section{METHODOLOGY}

\section{Experimental Setup}

The number of blades per row of the compressor under investigation are listed in Table 1. The impeller is an open type wheel and the design flow coefficient is $\Phi=0.15$. The compressor is driven by an electric motor, which gives the possibility to freely adjust the rotational speed or to run speed ramps. In all investigations presented in this paper the IVG angle was set to $0^{\circ}$, which means that the inflow is swirl free.

Table 1. Number of blades

\begin{tabular}{r|r} 
Row & \# of blades \\
\hline Inlet Guide Vane & 11 \\
Impeller & 16 \\
Diffuser & 19
\end{tabular}

The test rig is equipped with a standard setup of measurement techniques to acquire the operating point and the characteristic of the compressor stage. Additionally, measurement techniques specially for the investigation of forced response are installed in the compressor. To capture the vibration amplitudes, strain gauges are applied on five blades of the impeller. As visible in Figure 1, the positions of the strain gauges are equal on each blade to identify blade to blade variations of the vibration amplitude. The distribution of the instrumented blades is given in Figure 5.

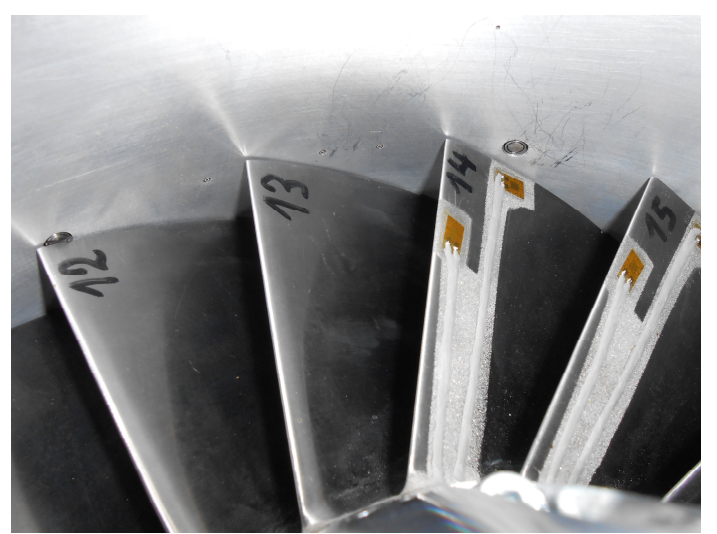

Figure 1. Impeller with mounted strain gauges. In the casing the tip timing probes are visible.

To transfer the data from the rotating impeller to the stationary casing, a digital telemetry is installed. Additionally, a non-intrusive tip timing system is flush-mounted in the shroud to identify the nodal diameter of the vibration. The arrangement of both systems is chosen to capture two distinct modes very well. Figure 1 shows the compressor wheel with mounted strain gauges and tip timing probes above blades numbers 12 and 14 in the shroud.

For the cross-comparisons the data measured by the strain gauges and the tip-timing probes were converted into the 
maximum von Mises Stress in the blade. The conversion factors were determined from FE calculations. Then, the numerically calculated maximum alternating stresses can be validated against the derived maximum stresses. The validity of the numerically predicted flow field is a key feature for the correct estimation of forcing and damping. Within this paper the simulated compressor characteristic is compared with the measured compressor map to ensure the validity of the numerical setup (c.f. Figure 7).

A pre-test investigation of the impeller's vibration characteristic at the TU Cottbus (Kühhorn \& Beirow, 2010) showed the mistuning distribution of the impeller blades eigenfrequencies at rest. A more detailed description will be given in the results and discussion section (c.f. Figure 5).

\section{Numerical Setup}

To predict the static and alternating stresses occurring in a centrifugal impeller during resonance, a mechanical and an aerodynamic model must be analyzed. Within this investigation, the mechanical simulations are performed in ANSYS MECHANICAL 19 and the aerodynamic simulations in NUMECA FineTurbo v12.2. The data exchange between both applications was carried out via in-house Python scripts. The whole tool chain is implemented as one-way coupling. Figure 2 shows the process scheme of the tool chain.
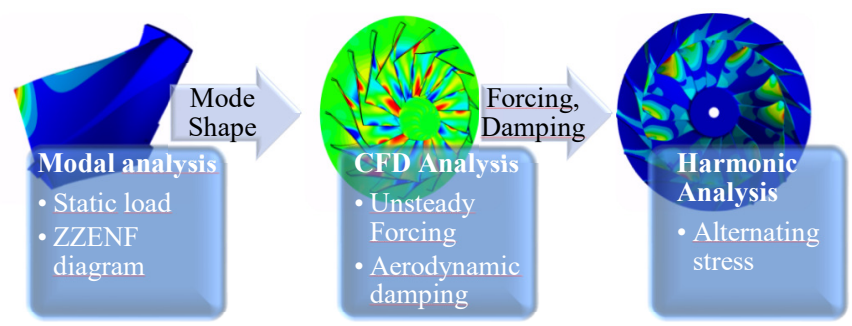

Figure 2. Schematic workflow of the one-way tool chain.

The mechanical analysis starts with a static load computation, which considers the centrifugal forces due to rotation. Superimposed on this solution, a pre-stressed modal analysis is carried out. A result of this calculation is the ' $\mathrm{Zig}$ Zag Engine-order Nodal-diameter Frequency' diagram (ZZENF), which plots the dispersion of the eigenfrequencies over the nodal diameter.

The ZZENF-diagram for the impeller under investigation is shown in Figure 3. The diffuser (yellow line) can excite the nodal diameter 3 and the IGV (green line) the nodal diameter 5. As the rotational speed can be chosen independently within a certain speed range, the first six modes could be excited by the diffuser (EO19) and the first two modes by the IGV (EO11). Within this paper the marked crossings with the eigenfrequency of mode 2 at approximately $3000 \mathrm{~Hz}$ are analyzed in detail. The numerical predicted rotational speed of the crossing with EO11 is $16^{\prime} 238 \mathrm{rpm}$, which is only $3 \%$ lower than the design speed. The crossing with EO19 occurs at a rotational speed of only 9'355 rpm.

The mode shape of the vibration is shown in Figure 4. As the ZZENF diagram indicates, this mode is a blade mode with nearly no vibration of the hub disc. The eigenfrequencies of the mode are only slightly changing with nodal diameter,

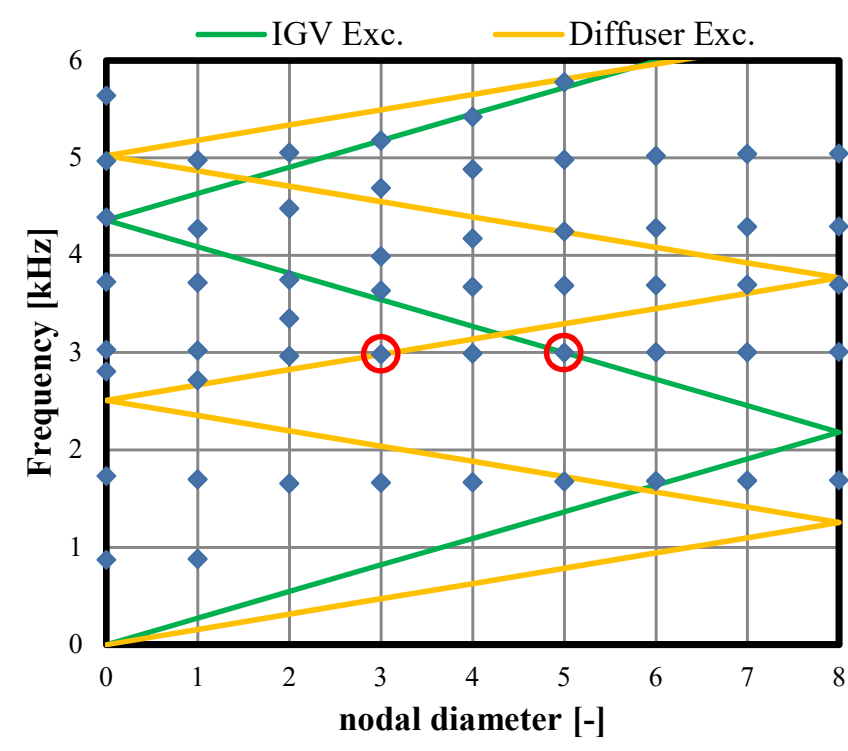

Figure 3. Numerically predicted ZZENF diagram of the impeller with excitation lines of the IGV and the diffuser and marked resonances $(\circ)$.

which suggests a weak coupling of the blades and the hub disc. The local deflections of the blade are phase shifted against each other. In a time sequence the maximum deflections of the two vibration antinodes would occur at different times. In the frequency domain the deflection becomes complex. The real and imaginary parts of the mode shape are exported and processed by a Python script for the import into FineTurbo. Thereby, only the vibration of the blade itself is transferred into the CFD simulation. In a case with significant movements of the hub, the vibration of the whole wetted surface must be exchanged (Walton \& Tan, 2015).

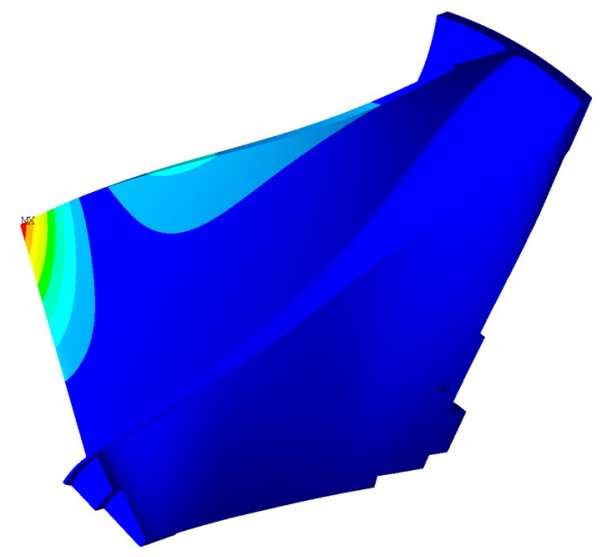

Figure 4. Mode shape of the investigated mode 2 at approximately $3000 \mathrm{~Hz}$.

The CFD simulations were performed with the fluidstructure module and the NLH approach implemented in FineTurbo. The complex mode shape has to be imported and interpolated from the FE mesh onto the CFD mesh. This is done by the 'Inverse Distance Weight' function provided by the fluid-structure module. The blunt trailing edge of the impeller prevents the meshing of the fillet radii's real shape in the CFD mesh. In the FE model the fillet radii must be modelled. Thus, only the vibration of the blade part above the fillet is transferred to the one-way coupled aeroelastic CFD simulation. But, as the blade only bends above the fillet radius 
(c.f. Figure 4), the mode shape is not affected by this inequality of the computational domains. The static deflection of the blade due to centrifugal forces was not considered.

In the NLH setup the Rank-2 method was chosen, so that the influence of the stator rows on each other is modelled. The frequencies of Tyler-Sofrin modes (e.g. (König, et al., 2009)) were not considered. A preliminary study of the compressor only showed very little amplitudes of these frequencies. Table 2 lists the frequencies, which are resolved within the NLH calculations.

\begin{tabular}{|c|c|c|c|}
\hline & $I G V$ & Impeller & Diffuser \\
\hline EO16 & H1 & - & $\mathrm{H} 1, \mathrm{H} 2, \mathrm{H} 3$ \\
\hline E011 & - & $\mathrm{H} 1, \mathrm{H} 2, \mathrm{H} 3$ & - \\
\hline Structure & - & $\mathrm{H} 1$ & - \\
\hline EO19 & - & $\mathrm{H} 1, \mathrm{H} 2, \mathrm{H} 3$ & - \\
\hline
\end{tabular}

In the aeroelastic simulations a one-way coupling was used. The maximum deflection of the mode shape, as well as the frequency and the nodal diameter of the vibration are user inputs. To limit the mesh distortion during the vibration, the maximum deflection was set to $100 \mu \mathrm{m}$. The frequency and the nodal diameter are set to the corresponding values of the resonance under investigation.

As stated above, two resonance crossings with mode 2 were examined. Within this paper the results of a swirl free inlet flow are presented and discussed. The influence of preswirl, which has an important influence on the alternating stresses, was also investigated at the test rig, but is part of ongoing research. Three operating points on each speed line (choke, maximum efficiency and near surge) were simulated with blade vibration. Further information can be found in the CFD results section.

The results of the simulations were evaluated with focus on the aerodynamic excitation and damping. For both analyses, the calculation of the aerodynamic work is essential. Due to the resonant condition, the frequencies of the pressure fields induced by the excitation and the vibration should be identical. But, although considering all frequencies in the same simulation, very small numerical rounding errors let the flow solver treat both fields separately. It was investigated, if these two flow fields interact with each other. A simulation with switched off excitation frequencies was conducted. In comparison to the simulation with all frequencies, the resulting aerodynamic damping did not change. Therefore, a parted analysis of the excitation and damping flow field from the simulation setup described in Table 2 is possible. The aerodynamic work

$$
W=\int_{0}^{T} \oint_{A}(\overline{\bar{\sigma}} \cdot \underline{n}) \cdot \underline{\dot{s}} d A d t
$$

can, if the amount of the shear stresses is neglected, be expanded and - assuming harmonic cosine oscillations integrated to

$$
\begin{gathered}
W=\pi \cdot \oint_{A} \hat{p} \cdot\left\{s_{x} n_{x} \sin \left(\varphi_{p}-\varphi_{x}\right)+s_{y} n_{y} \sin \left(\varphi_{p}-\right.\right. \\
\left.\left.\varphi_{y}\right)+s_{z} n_{z} \sin \left(\varphi_{p}-\varphi_{z}\right)\right\} d A
\end{gathered}
$$

From equation (3) it becomes clear that not only the product of the local amplitudes of the pressure fluctuation $\hat{p}$ and the deflection in cartesian coordinates $s_{x, y, z}$ determines the aerodynamic work. Also, the phasing between these two oscillating magnitudes plays an important role.

For the assessment of the aerodynamic damping, the aerodynamic work can be used to calculate the logarithmic decrement

$$
\Lambda=\frac{-W}{\|\underline{s}\|_{\max }^{2} m(2 \pi f)^{2}} .
$$

In the harmonic analysis of the structure this logarithmic decrement is imprinted as a global damping coefficient.

In contrast to that, the calculation of the aerodynamic forcing is not that straight forward. The phase differences of the unsteady pressure field and the blade displacement are decoupled in a one-way aeroelastic calculation. The phasing of the vibration can be chosen arbitrarily and the phasing of the unsteady pressure field, which is exciting the blade, depends on the spatial position of both blade rows in the CFD model. As indicated by equation (3), the phase difference between the vibration and the excitation force has a great influence on the resulting aerodynamic excitation work. During the postprocess the correct phase relationship can be reconstructed. Assuming that a linear superposition of the flow fields is possible, the phasing of the blade vibration can be altered until the aerodynamic work is maximal.

The magnitudes of the aerodynamic excitation work and the aerodynamic damping work cannot be compared directly. The excitation scales linearly and the aerodynamic damping work quadratically with the maximum deflection. Both works could only be compared, if the deflection is correct, but the deflection is chosen arbitrarily. The aerodynamic excitation work can be used to illustrate, if an unsteady pressure field is able to excite a certain mode shape.

For the calculation of the alternating stresses, the absolute values of the unsteady pressure field are imported into the mechanical harmonic analysis of the impeller and mapped onto the FE mesh. In combination with the damping coefficient, the resulting vibration strain and stress can be computed within a pre-stressed modal superposition analysis. 


\section{RESULTS AND DISCUSSION}

\section{Pre-test experimental results}

As outlined in the methodology section, several experimental investigations were conducted with the nonrotating impeller. The two main findings are reported in the following. Firstly, a mistuning measurement was performed.

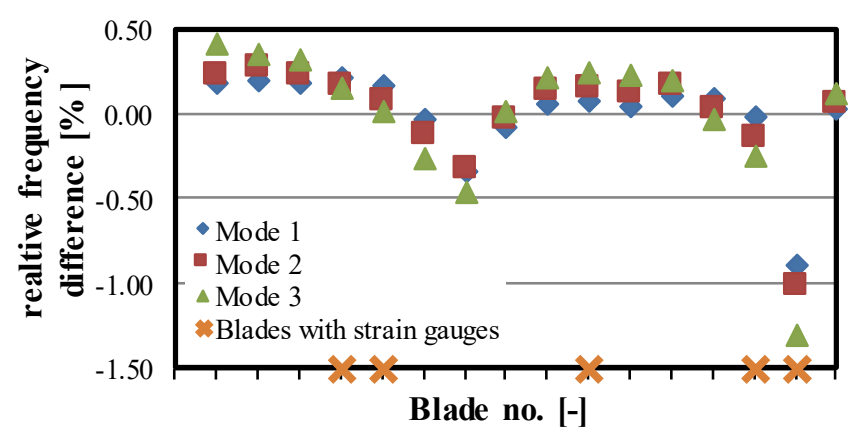

Figure 5. Mistuning analysis of the impeller, visualized by the blade's individual frequency deviation from the mean frequency of all blades.

From Figure 5 it gets obvious, that the impeller has a wavy mistuning distribution, which could be a result of the manufacturing strategy. A very similar distribution of the eigenfrequencies was visible in the tip timing measurement data. Actually, it was found out later, that the supposed mistuned blade with approximately 1 to $1.5 \%$ lower eigenfrequencies was the blade which fits best to the CAD model. As this CAD model was used for the investigation, the experimental and the numerical resonant frequencies differ. A further extended mistuning analysis is not content of this paper.

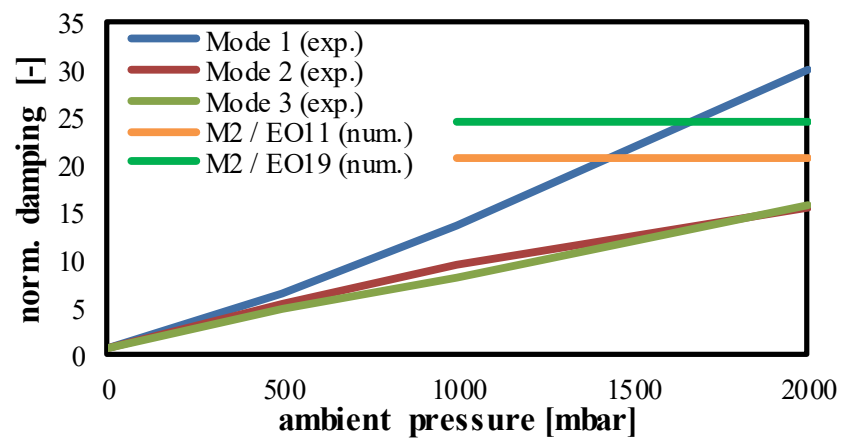

Figure 6. Measured aerodynamic damping at the static impeller at different pressure level and numerically predicted aerodynamic damping under rotation for the two investigated resonances.

Secondly, the aerodynamic damping at the non-rotating impeller at different ambient pressure level was measured by exciting the impeller with a modal hammer (Kühhorn \& Beirow, 2010). Figure 6 gives an overview of the final results. All damping values are normalized with the mean damping at technical vacuum (1mbar), which nearly represents the material damping. The results are in good accordance with other experimental investigations, e.g. (Zemp, et al., 2011). As stated above, Figure 6 shows that the aerodynamic damping is approximately one order of magnitude larger than the material damping. The magnitude of the damping values calculated by the presented tool chain are in good agreement with the measured values, although the measurements were conducted at the stationary impeller.

\section{Experimental results}

The measurements with the strain gauges and the tip timing probes were conducted during compressor speed sweeps. Thereby, the system characteristic was adjusted so that the compressor was operating a) at choke condition, b) approximately at maximum efficiency and c) at surge. Figure 7 shows the measured compressor characteristic with the three operating lines "Choke OL", "max. Eff. OL" and "Surge OL". Within this paper six resonant crossings are analyzed, which are highlighted by the red symbols.

Additionally, the corresponding six CFD points at which the numerical $\mathrm{HCF}$ analysis were performed are marked with blue symbols. By means of the marked resonant points, it can be seen that the numerically investigated resonance points occur at lower pressure ratios than the measured ones. This is due to the fact, that the basic CAD model represents the blade with the lowest eigenfrequencies (c.f. Figure 5). Thus, the first 3 numerically predicted eigenfrequencies are lower than the average measured frequencies of the structure. Accordingly, the rotational speeds at which the numerical resonances occur are lower than the measured ones.

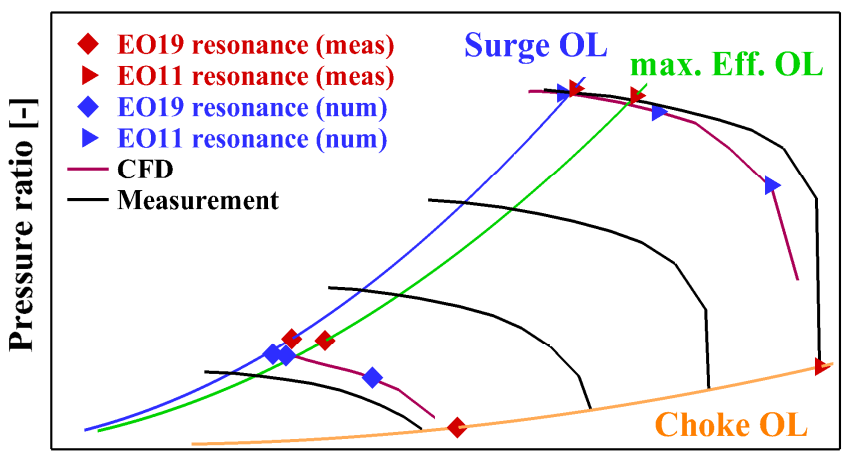

Volumeflow $\left[\mathrm{m}^{3} / \mathrm{s}\right]$

Figure 7. Measured compressor characteristic, measured resonance points $(\diamond, \downarrow)$, the three operating lines and the numerically investigated resonance points $(\diamond, \downarrow)$. The numerically predicted speed lines of the resonance speeds do not correspond to the shown speed lines.

The numerical choke operating points do not lie on the "Choke OL". Due to the onset of large separations in the vaned diffuser, the CFD calculations at choke conditions were unstable. A reasonable evaluation of the FSI and HCF behavior was not possible. Therefore, the numerically last stable operating points were chosen for this investigation. At both resonances, the mode 2 (c.f. Figure 4) is stimulated. The excitation by EO19 is induced by the upstream potential field of the vaned diffuser. The EO11 excitation is caused by the downstream wake of the IGV.

Figure 8 shows the mean measured alternating stresses at the resonance M2/EO19 for the three different operating lines. Additionally, the black bars indicate the range of variation of the different measurement techniques, also including 
mistuning effects. In advance of the numerical results, both figures show the results of the developed tool chain. The measured stress levels are very low, but it was possible to clearly identify the amplitude magnifications.

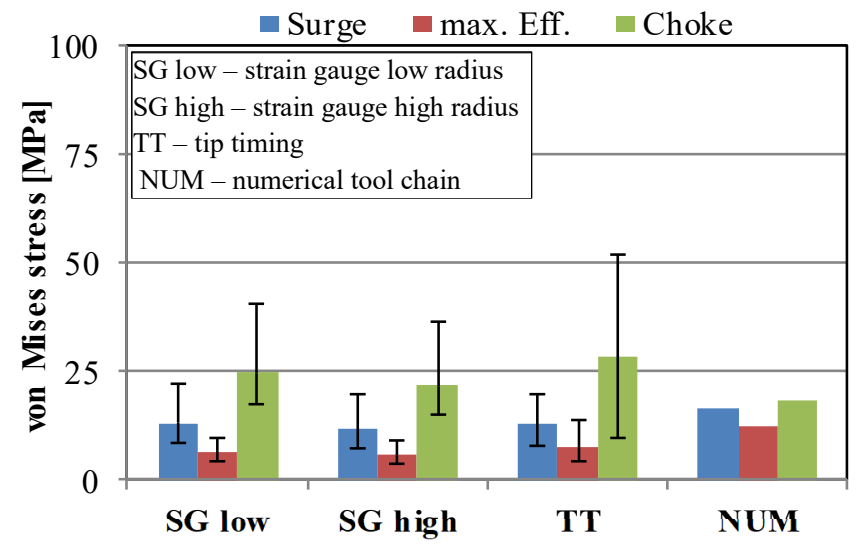

Figure 8. Measured and predicted alternating stress @ M2/EO19 resonance. The black bars indicate the range of measured variations.

All three measurements results exhibit similar trends: The absolute level as well as the growth across the speed line of the mean alternating stress are qualitatively coinciding. Especially, the accordance of the tip timing and the strain gauge measurements is good. This suggests that both measurement techniques are suitable for the investigation of resonance stresses in centrifugal compressors. Especially low frequency modes, which, due to the low blade numbers in centrifugal compressors, are most endangered to be stimulated, can be captured well by the tip timing system. These similarities are confirmed by the measurements of the M2/EO11 resonance, which are plotted in Figure 9.

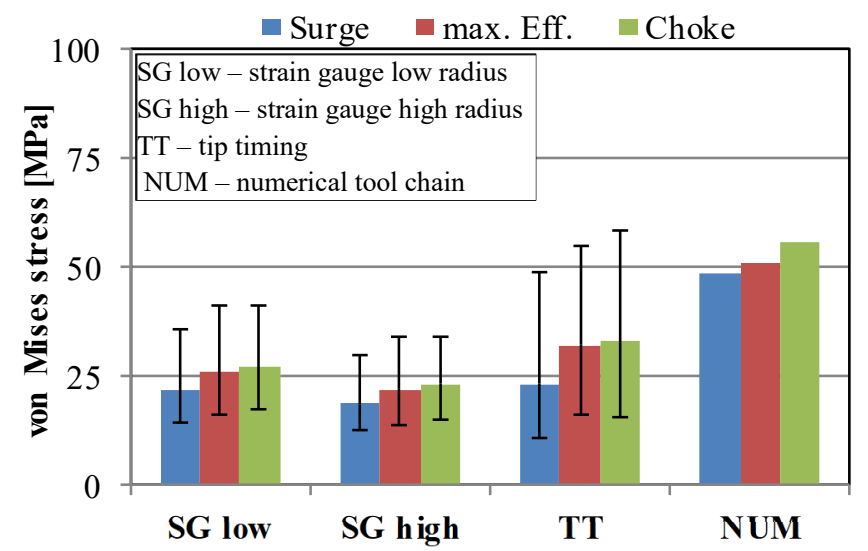

Figure 9. Measured and predicted alternating stress @ M2/EO11 resonance. The black bars indicate the range of measured variations.

The measured alternating stresses are very low for both resonant cases. The impeller is not suffering any HCF damage. During the other resonance crossings with an IGV angle of $0^{\circ}$, which are not presented in this paper, the measured alternating stresses are lower than $60 \mathrm{MPa}$. Therefore, it can be stated that for the swirl free inlet condition the compressor is $\mathrm{HCF}$ safe.

The resonant excitation by the diffuser (M2/EO19) causes the lowest alternating stresses at maximum efficiency flow conditions (c.f. Figure 8). Towards the operating boundaries, the stresses are increasing, whereby the choke condition causes the highest alternating stress level. The only parameters, which are changing between these three resonance conditions are the aerodynamic flow conditions. As a first estimation, which will be confirmed later for this case (c.f. Figure 10), the aerodynamic damping is assumed to be nearly constant. It follows that the aerodynamic excitation must change. In the "max. Eff." operating point the flow incidence at the leading edge of the diffuser is minimal. Thus, the potential field of the diffuser is smallest in this operating point and in conclusion the excitation is low. The flow incidence at the diffuser leading edge is grater in the "choke" than in the "surge" operating point. Consequently, the measured alternating stresses are largest at choke. Generally, the pressure fluctuations caused by the diffuser are greatest at the trailing edge of the impeller. As the mode shape (c.f. Figure 4) has nearly no deflection in the rear part of the blade, these pressure fluctuations are not able to stimulate the mode 2 into any critical vibration.

In contrast to that, the pressure fluctuations induced by the IGV are mainly acting on the front part of the impeller. In this area the mode 2 has the largest deflections at the blade tip. The mode shape can therefore be stimulated more easily at the leading edge. Finally, this results in a higher alternating stress level for the M2/EO11 than for the M2/EO19 resonance.

Again, the largest alternating stresses are measured at choke (c.f. Figure 9). With increasing flow rate, the magnitude of the wake induced by the IGV and in conclusion the excitation force enlarges. Again, assuming a constant aerodynamic damping the highest alternating stresses occur at choke conditions.

\section{Numerical results}

Figure 8 and Figure 9 show the experimentally and numerically determined maximum alternating stresses within the impeller. The numerical results overestimate the alternating stresses. The results of the choke operating points are not taken into consideration. As shown in Figure 7, it was not possible to get stable numerical results at the choke conditions. Compared to the average of the corresponding measured alternating stresses the calculated alternating stress of the M2/EO19 crossing is $60 \%$ and of the M2/EO11 crossing is $110 \%$ higher. These deviations can have different reasons. First of all, the numerical investigated geometry differs from the real geometry (c.f. section pre-test experimental results) as it represents a completely tuned system. As indicated by the black bars in Figures 8 and 9, the measurements exhibit a distinct spread of approximately $60-150 \%$ of the average value. This behavior cannot be captured by a tuned system. Secondly, the numerical lower resonance speeds and the shift of the operation points lead to aerodynamical situations, which differ from the real situation within the machine. The influence of hysteresis effects and sweep rate were investigated experimentally and could be excluded as element of uncertainty. Considering all these uncertainties, the achieved results are very promising that the developed tool chain correctly captures the main FSI effects. Further 
investigations of the uncertainties are content of ongoing and future projects and thus, will not be presented in this paper.

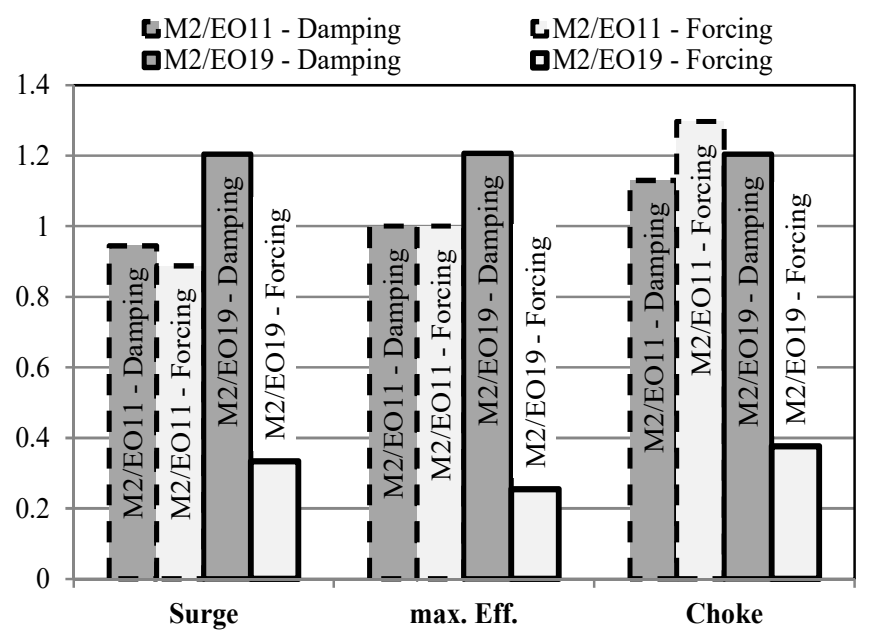

Figure 10. Aerodynamic excitation and damping values deduced from the CFD simulations for both resonances.

To get a deeper insight into the aerodynamic forcing and damping, Figure 10 shows the evaluated aerodynamic forces. The values are normalized with the values in the resonance M2/EO11 at the max. Eff. operating point. The grey shaded bars visualize the aerodynamic damping and the white shaded bars the aerodynamic excitation. Beginning with the aerodynamic excitation, the conclusions drawn in the experimental results section can be confirmed. Overall the EO19 excitation is approximately 2.5-4.0 times smaller than the EO11 excitation of mode 2. On the other hand, the aerodynamic damping of EO19 is higher than of EO11, which finally results in the very low alternating stress level. Obviously, the damping for the M2/EO19 resonance is nearly constant across the speed line. As the relative Mach number level is low on this speed line, the propagation of the pressure disturbances seems to be similar for all operation points. In contrast to that, the excitation slightly changes across the speed line, whereby the excitation is lowest in the max. eff. operating point. This can be explained by the potential field around the diffuser blade, which's upstream influence is smallest for this operating point and thus, the level of the induced pressure fluctuations on the impeller blade is low.

The main reason for the very low excitation by the diffuser gets obvious by analyzing the spatial distribution of the excitation work defined by equation (2) on the blade surface. Figure 11 shows a cut on $90 \%$ span for the choke and surge operating points, whereby the M2/EO19 excitation is inked in red and purple and the M2/EO11 excitation in green and orange. Additionally, the total deformation is indicated and it gets very clear that only areas of large deflection contribute to the total excitation work. As Figure 11 indicates, the excitation work performed by the diffuser is very low. The main excitation work is done at the leading edge. In the midsection of the blade even damping occurs, visibly by the negative work. For both M2/EO19 operating points, the shape of the work distribution is very similar and likewise, according to Figure 10, the global excitation is.
The aerodynamic excitation caused by the IGV is monotonically rising from surge to choke. This can be attributed to the increasing flowrate and the thereby enlarged wake of the IGV. According to Figure 11, the excitation at the leading edge dominates. The flow incidence angle at the leading edged changes with the flow rate. This entails that the largest amounts of the excitation work are flipping from the suction side at surge to the pressure side at choke.

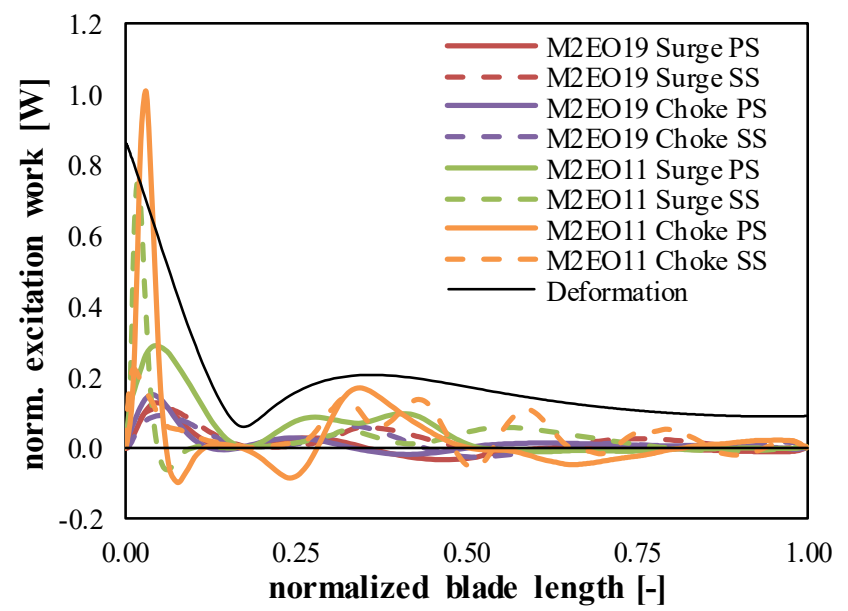

Figure 11. Normalized excitation work due to the diffuser potential field and the IGV wake on the blade surface at the $90 \%$ span cut.

Finally, the aerodynamic damping is analyzed in a similar way as the excitation. In Figure 12 the work done by the unsteady pressure field, which comes about the vibration of the blade, is plotted on the $90 \%$ span cut. For both engine orders, locally the work is positive, which indicates areas of excitation. If these work contributions would be greater than the negative ones, the blade would flutter. The summed up total work is negative in all cases, so the vibration is always damped.

The damping work of the M2/EO19 resonance is very similar for both operating points. This confirms the suggestion, that in both cases the pressure fluctuations caused by the vibration are convected likewise. In the M2/EO11 resonance the area of greatest damping switches from the suction side at surge to the pressure side at choke. The differences of the flow conditions at the leading edge between the surge and the choke operating point are clearer. Especially, a distinct area of transonic flow condition, but no shock wave, emerges at choke, which drastically changes the propagation of the pressure disturbances induced by the vibration of the blade. These strong interactions between the flow field and the pressure fluctuations make it difficult to derive any generally valid dependencies.

In the introduction it was referred to the partially high level of tailor-made geometries, which are derived from a baseline geometry. Therefore, every resonance occurring in a machine design has to be investigated individually regarding their HCF characteristic. 


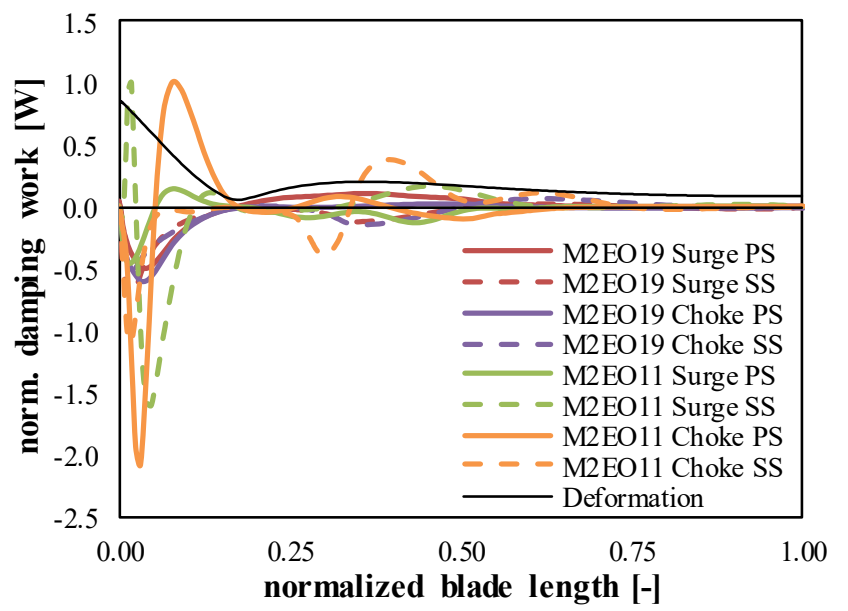

Figure 12. Normalized damping work caused by the unsteady pressure field of the blade vibration on the blade surface at the $90 \%$ span cut.

\section{CONCLUSIONS}

Within this paper measured and numerically calculated alternating stresses during resonance in an impeller of an industrial centrifugal compressor were shown and analyzed. Both parts of the investigation focus on the second eigenmode of the impeller, which has an eigenfrequency of approximately $3000 \mathrm{~Hz}$. This mode can be stimulated by the IGV as well as by the diffuser.

The alternating stresses measured by two independent measuring devices during resonance are all in good accordance with each other. This guarantees a good quality of the measurement results and enables a comparison to numerical results. For future projects it could be contemplated to reduce the measurement effort to only one technique. Summarized, the measured alternating stresses with an IGV angle of $0^{\circ}$ are far below any critical values for all resonances. The impeller is HCF safe.

Pre-test investigations exhibited a distinct mistuning characteristic of the impeller. It was emphasized that the discussed numerical results were generated with a tuned system, which was constructed with the blade geometry, which has the lowest eigenfrequencies. Therefore, a complete match between the numerical results and the measurements cannot be expected.

The comparison of the numerical and the average experimental maximum alternating stresses showed deviations of approximately $60 \%$ for the diffuser excitation and $110 \%$ for the IGV excitation. These deviations are too high for a reliable statement about the long-life fatigue strength. On the other hand, it must be taken into account that the measured alternating stresses are varying from $60 \%$ to $150 \%$ of the average stress from blade to blade. Further investigations regarding the uncertainties between the numerical model and the real compressor have to be carried out to get a better evaluation of the gained numerical results.
The results of the tool chain provide a good first indication about the HCF risk of a resonant crossing.

Encouragingly, the numerical results show the same trends along a speed line as the measurements do. For the simulation of the unsteady flow, a frequency domain formulation (NLH) was used. The investigation shows, that this numerical setup is able to correctly capture the qualitative trends of the unsteady flow fields. Especially, the verification of the simulations with moving meshes is a great progress towards a fast and reliable evaluation of HCF induced by rotor-stator interactions.

Finally, it can be stated that the developed tool chain for the assessment of HCF is able to reproduce the measured finding for one particular mode within a distinct uncertainty. To be able to generalize this statement further comparisons with the measurement results of other resonances have to be conducted. Further on, the influence of the blade geometry on the calculated alternating stresses has to be clarified. These issues are content of ongoing research.

\section{NOMENCLATURE}

\begin{tabular}{lll}
\multicolumn{3}{l}{ Formula symbols } \\
$\Phi$ & {$[-]$} & Flow coefficient $4 \dot{m} /\left(\pi \rho_{0, t} u_{2} d_{2}^{2}\right)$ \\
$\varphi$ & {$\left[{ }^{\circ}\right]$} & Phase angle \\
$\mathrm{W}$ & {$[\mathrm{J}]$} & Work \\
$\mathrm{n}$ & {$[-]$} & Normal direction \\
$\sigma$ & {$\left[\mathrm{N} / \mathrm{m}^{2}\right]$} & Stress tensor \\
$\mathrm{s}$ & {$[\mathrm{m}]$} & Deflection \\
$\mathrm{A}$ & {$\left[\mathrm{m}^{2}\right]$} & Area \\
$\mathrm{t}$ & {$[\mathrm{s}]$} & Time \\
$\mathrm{T}$ & {$[\mathrm{s}]$} & Time period $=1 / \mathrm{f}$ \\
$\mathrm{f}$ & {$[1 / \mathrm{s}]$} & Frequency \\
$\mathrm{p}$ & {$[\mathrm{Pa}]$} & Pressure \\
$\pi$ & {$[-]$} & Pi \\
$\Lambda$ & {$[-]$} & Logarithmic decrement \\
$\mathrm{m}$ & {$[\mathrm{kg}]$} & Modal mass \\
$\dot{m}$ & {$[\mathrm{~kg} / \mathrm{s}]$} & Massflow \\
$\rho_{0, t}$ & {$\left[\mathrm{~kg} / \mathrm{m}^{3}\right]$} & Total density @ inlet \\
$u_{2}$ & {$[\mathrm{~m} / \mathrm{s}]$} & Peripheral velocity @ impeller outlet \\
$d_{2}$ & {$[\mathrm{~m}]$} & Impeller outlet diameter
\end{tabular}

\section{Indices}

$\begin{array}{ll}\mathrm{x}, \mathrm{y}, \mathrm{z} & \text { Cartesian direction } \\ \mathrm{P} & \text { pressure }\end{array}$

\section{Accents}

$\begin{array}{cl}\circ & \text { Vector } \\ \overline{\hat{o}} & \text { Amplitude } \\ \overline{\bar{o}} & \text { Tensor } \\ \|\circ\| & \text { Absolute value }\end{array}$




\section{ACKNOWLEDGMENTS}

The authors would like to thank the MAN Energy Solutions SE for supporting this study and giving the permission to publish the results.

The work was conducted as a part of the funding program AG Turbo of the German Federal Ministry of Economics and Technology (BMWi) under the name Cooreflex-turbo 1.3.2 'Fluid-Structure-Interaction in Radial and Axial Compressors' (03ET7020N).

\section{REFERENCES}

Chen, X. et al., 2017. Research on Failure of Semi-Open Centrifugal Impeller Under Aerodynamic Load. ASME Turbo Expo: Power for Land, Sea, and Air, Volume 7B: Structures and Dynamics, p. V07BT36A017.

Debrabandere, F., Tartinville, B., Hirsch, C. \& Coussement, G., 2012. Fluid-Structure Interaction Using a Modal Approach. Journal of Turbomachinery, 6, Volume 134, pp. 051043--051043-6.

Debrabandere, F., Tartinville, B., Hirsch, C. \& Coussement, G., 2013. Extension of the non-linear harmonic method to flow computations around moving and deforming structures. 1.

Dickmann, H.-P.et al., 2005. Unsteady Flow in a Turbocharger Centrifugal Compressor: Three-Dimensional Computational Fluid Dynamics Simulation and Numerical and Experimental Analysis of Impeller Blade Vibration. Journal of Turbomachinery, 2, Volume 128, pp. 455-465.

Dickmann, H.-P.et al., 2009. Unsteady Flow in a Turbocharger Centrifugal Compressor: 3D-CFD Simulation, Impeller Blade Vibration and Vaned Diffuser-Volute Interaction. ASME Turbo Expo: Power for Land, Sea, and Air, Volume 7: Turbomachinery, Parts A and B, pp. 1471-1485. Elder, R. et al., 2013. Investigation of Efficient CFD Methods for the Prediction of Blade Damping. ASME Turbo Expo: Power for Land, Sea, and Air, Volume 7B: Structures and Dynamics, p. V07BT33A009.

Fruth, F., Jeschke, P. \& Franz, H., 2016. On the Scaling of Aeroelastic Parameters for High Pressure Applications in Centrifugal Compressors. Turbo Expo: Power for Land, Sea, and Air, p. V07BT34A019.

Jenny, P. \& Bidaut, Y., 2016. Experimental Determination of Mechanical Stress Induced by Rotating Stall in Unshrouded Impellers of Centrifugal Compressors. Journal of Turbomachinery, 12, Volume 139, pp. 031011--031011-10.

Kammerer, A. \& Abhari, R. S., 2010. Blade Forcing Function and Aerodynamic Work Measurements in a High Speed Centrifugal Compressor With Inlet Distortion. Journal of Engineering for Gas Turbines and Power, 6, Volume 132, pp. 092504--092504-11.

Kammerer, A. \& Abhari, R. S., 2010. The Cumulative Effects of Forcing Function, Damping, and Mistuning on Blade Forced Response in a High Speed Centrifugal Compressor With Inlet Distortion. Journal of Engineering for Gas Turbines and Power, 8, Volume 132, pp. 122505--122505-10. König, S., Petry, N. \& Wagner, N. G., 2009. Aeroacoustic Phenomena in High-Pressure Centrifugal Compressors - a
Possible Root Cause for Impeller Failures. Proceedings of the Thirty-Eighth Turbomachinery Symposium, pp. 103-122.

Kühhorn, A. \& Beirow, B., 2010. Method for Determining Blade Mistuning on Integrally Manufactured Rotor Wheels. US, Patent No. 2010/0286934 A1.

Lerche, A. H., Moore, J. J., White, N. M. \& Hardin, J., 2012. Dynamic Stress Prediction in Centrifugal Compressor Blades Using Fluid Structure Interaction. Turbo Expo: Power for Land, Sea, and Air, pp. 191-200.

Mischo, B. et al., 2018. Numerical and experimental fsi-study to determine mechanical stresses induced by rotating stall in unshrouded centrifugal compressor impellers. Turbo Expo: Power for Land, Sea, and Air.

Platzer, M. \& Carta, F., 1988. AGARD manual on aeroelasticity in axial-flow turbomachines. Volume 2: Structural dynamics and aeroelasticity, s.l.: s.n.

Seinturier, E., 2007. Forced response computation for bladed disks industrial practices and advanced methods. 12th IFToMM World Congress, Besancon, France, June 18-21, 2007, 9.

Seinturier, E. et al., 2004. Forced Response Prediction: Methodology for the Design of HP Compressors Bladed Disks. ASME Turbo Expo: Power for Land, Sea, and Air, Volume 6: Turbo Expo 2004, pp. 317-326.

Srinivasan, A. V., 1997. Flutter and Resonant Vibration Characteristics of Engine Blades: An IGTI Scholar Paper. ASME. Turbo Expo: Power for Land, Sea, and Air, Volume 4: Manufacturing Materials and Metallurgy; Ceramics; Structures and Dynamics; Controls, Diagnostics and Instrumentation; Education; IGTI Scholar Award, p. V004T17A001.

Wagner, N. G., Winkes, G., König, S. \& Süß, S., 2011. OnSite Determination of the Control Line for Integrally Geared Compressors for Avoidance of Impeller Fatigues. Proceedings of the First Middle East Turbomachinery Symposium, Feburary 13-16, 2011, Doha, Qatar.

Walton, E. J. \& Tan, C. S., 2015. Forced Response of a Centrifugal Compressor Stage due to the Impeller-Diffuser Interaction. ASME Turbo Expo: Power for Land, Sea, and Air, Volume 2C: Turbomachinery, p. V02CT42A001.

Zemp, A. \& Abhari, R. S., 2012. Vaned Diffuser Induced Impeller Blade Vibrations in a High-Speed Centrifugal Compressor. Journal of Turbomachinery, 11, Volume 135, pp. 021015--021015-9.

Zemp, A., Abhari, R. S. \& Ribi, B., 2011. Experimental Investigation of Forced Response Impeller Blade Vibration in a Centrifugal Compressor With Variable Inlet Guide Vanes: Part 1--Blade Damping. ASME Turbo Expo: Power for Land, Sea, and Air, Volume 6: Structures and Dynamics, Parts A and $B$, pp. 1369-1380.

Zemp, A., Abhari, R. S. \& Schleer, M., 2011. Experimental Investigation of Forced Response Impeller Blade Vibration in a Centrifugal Compressor With Variable Inlet Guide Vanes: Part 2--Forcing Function and FSI Computations. ASME Turbo Expo: Power for Land, Sea, and Air, Volume 6: Structures and Dynamics, Parts A and B, pp. 1381-1392. 